\title{
Programme familial d'entrainement aux habiletés pour les parents ayant une dépendance à l'alcool ou aux drogues et leurs enfants âgés de 6 à 12 ans : caractéristiques des familles rejointes par le programme Parenting training program for parents with an addiction and their children aged 6 to 12: Characteristics of families reached by the program
}

\author{
Myriam Laventure, Francine Ferland, Nadine Blanchette-Martin, Rosalie \\ Genois, Pascal Garceau et Stéphane Turcotte
}

Volume 47, numéro 2, 2018

URI : https://id.erudit.org/iderudit/1054063ar

DOI : https://doi.org/10.7202/1054063ar

Aller au sommaire du numéro

Éditeur(s)

Revue de Psychoéducation

ISSN

1713-1782 (imprimé)

2371-6053 (numérique)

Découvrir la revue

Citer cet article

Laventure, M., Ferland, F., Blanchette-Martin, N., Genois, R., Garceau, P. \& Turcotte, S. (2018). Programme familial d'entrainement aux habiletés pour les parents ayant une dépendance à l'alcool ou aux drogues et leurs enfants âgés de 6 à 12 ans : caractéristiques des familles rejointes par le programme. Revue de psychoéducation, 47(2), 313-332. https://doi.org/10.7202/1054063ar
Résumé de l'article

La présence d'une dépendance à l'alcool ou aux drogues chez un parent a des impacts sur l'unité familiale et son fonctionnement. Elle peut influencer les compétences parentales des parents et grandement affecter le développement des enfants. Au Québec, depuis 2008, deux programmes d'entrainement aux habiletés parentales créés spécifiquement pour les enfants de 6-12 ans dont un des parents a une dépendance (PEHP-Dépendance) ont été implantés dans certains centres de réadaptation en dépendance (CRD). La présente étude vise à identifier les caractéristiques des familles qui ont participé à un PEHP-Dépendance en CRD au Québec et vérifier si ces familles représentent un groupe homogène. L'échantillon est composé de 80 familles et de 108 enfants ayant participé au PEHP-Dépendance. Les résultats indiquent que les familles inscrites aux PEHP-Dépendance présentent des caractéristiques comparables à celles habituellement associées aux familles où la dépendance à l'alcool et aux drogues, et qu'elles pourraient donc bénéficier de ce genre d'intervention. Quatre profils-types différents de familles basés sur les difficultés familiales et psychologiques des parents ont été identifiés, représentant un groupe de participants hétérogènes dans les PEHP-Dépendance à l'étude. Dans le processus d'implantation de PEHP spécifique aux familles dont les parents ont une dépendance à l'alcool et aux drogues, ces résultats sont une première étape permettant de mieux adapter les interventions.
Tous droits réservés ( $\odot$ La Corporation de la Revue Canadienne de Psycho-Éducation, 2018
Ce document est protégé par la loi sur le droit d'auteur. L'utilisation des services d’Érudit (y compris la reproduction) est assujettie à sa politique d'utilisation que vous pouvez consulter en ligne.

https://apropos.erudit.org/fr/usagers/politique-dutilisation/ 


\section{Programme familial d'entrainement aux habiletés pour les parents ayant une dépendance à l'alcool ou aux drogues et leurs enfants âgés de 6 à 12 ans: Caractéristiques des familles rejointes par le programme}

\section{Parenting training program for parents with an addiction and their children aged 6 to 12: Characteristics of families reached by the program}

\section{Laventure ${ }^{1,3}$ \\ F. Ferland ${ }^{2}$ \\ N. Blanchette- \\ Martin ${ }^{2,3}$ \\ R. Genois ${ }^{2}$ \\ P. Garceau ${ }^{2}$ \\ S. Turcotte ${ }^{4}$}

1 Université de Sherbrooke

2 Service de recherche en dépendance CIUSSSCN/ CISSSCA

3 Recherche et intervention sur les substances psychoactives Québec (RISQ)

4 Centre intégré de santé et de services sociaux de ChaudièreAppalaches

\section{REMERCIEMENTS}

La rédaction de cet article a été rendue possible grâce à la contribution financière de l'équipe de recherche et intervention sur les substances psychoactives Québec (RISQ)

\section{Correspondance :}

Myriam Laventure

Université de Sherbrooke

Département de

psychoéducation

2500 boul. Université

Sherbrooke, QC J1K2R2

Tél. : 819-821-8000 \#62420

Myriam.Laventure@

USherbrooke.ca

\section{Résumé}

La présence d'une dépendance à l'alcool ou aux drogues chez un parent a des impacts sur l'unité familiale et son fonctionnement. Elle peut influencer les compétences parentales des parents et grandement affecter le développement des enfants. Au Québec, depuis 2008, deux programmes d'entrainement aux habiletés parentales créés spécifiquement pour les enfants de 6-12 ans dont un des parents a une dépendance (PEHP-Dépendance) ont été implantés dans certains centres de réadaptation en dépendance (CRD). La présente étude vise à identifier les caractéristiques des familles qui ont participé à un PEHP-Dépendance en CRD au Québec et vérifier si ces familles représentent un groupe homogène. L'échantillon est composé de 80 familles et de 108 enfants ayant participé au PEHP-Dépendance. Les résultats indiquent que les familles inscrites aux PEHP-Dépendance présentent des caractéristiques comparables à celles habituellement associées aux familles où la dépendance à l'alcool et aux drogues, et qu'elles pourraient donc bénéficier de ce genre d'intervention. Quatre profils-types différents de familles basés sur les difficultés familiales et psychologiques des parents ont été identifiés, représentant un groupe de participants hétérogènes dans les PEHPDépendance à l'étude. Dans le processus d'implantation de PEHP spécifique aux familles dont les parents ont une dépendance à l'alcool et aux drogues, ces résultats sont une première étape permettant de mieux adapter les interventions.

Mots-clés : Dépendance, alcool, drogues, programme familial, pratiques éducatives, évaluation d'implantation 


\begin{abstract}
The addiction to alcohol or psychoactive substances of a parent has impacts on the family unit and its functioning. It can influence parenting skills of the parents and greatly affect the children's development. In Quebec, since 2008, two parental management training programs created especially for children from 6-12 years old whose one parent has problems with substances (PEHP-Dependance) have been implemented in some addiction rehabilitation centers (CRD). This study aims to identify the characteristics of the families attending a PEHPDependence in CRD in Quebec and to investigate if these families represent a homogeneous group. The sample is composed of 80 families and 108 children who participated in the PEHP-dependence. The results show that these families share comparable characteristics with families typically affected by alcohol or psychoactive substances addiction, who could also benefit from this type of intervention. Four types of families have been identified based on familial difficulties and parent's psychological problems, representing a heterogeneous group of participants in PEHP-Dependance in the study. In the process of implantation of PEHP specific to families whose parents have an addition to alcohol and drugs, these results are a first step to better adapt interventions.
\end{abstract}

\title{
Keywords: addiction, dependence, alcohol, psychoactive substances, family func- tioning program, parenting, implantation evaluation
}

\section{Introduction}

La présence d'une dépendance à l'alcool et aux drogues chez un parent a des impacts sur le fonctionnement familial et représente un facteur de risque élevé quant aux compétences parentales des parents (Kumpfer et Bluth, 2004, Landry et al., 2010). La dépendance se définit par un mode d'utilisation inapproprié de l'alcool ou d'une drogue, entrainant une détresse ou un dysfonctionnement, incluant la présence de tolérance et la perte de contrôle (DSM-IV-TR, 2000). Plusieurs écrits rapportent des impacts importants de la dépendance sur l'unité familiale et son fonctionnement, entre autres, une diminution de la disponibilité physique et psychologique du parent pour répondre aux besoins de l'enfant (Barnard, 2006), une limitation importante de la disponibilité en temps, en argent et en énergie afin de répondre aux responsabilités professionnelles et familiales, une présence inconstante aux soins de l'enfant, un niveau inférieur de réponse aux besoins de l'enfant (Solis, Shadur, Burns et Hussong, 2013) ainsi qu'une diminution de la sensibilité parentale (Fraser, Harris-Britt, Thakkallapalli, Kurtz-Cortes et Martin, 2010; Soderström et Skårderud, 2013; Street, Whitlingum, Gibson, Cairns et Ellis, 2008).

Outre les impacts sur les compétences parentales, la présence d'une dépendance à l'alcool et aux drogues chez un parent influence le développement des enfants notamment en ce qui a trait au lien d'attachement qui affecte grandement la qualité du développement psychoaffectif de l'enfant (Bukatko et Daehler, 2004; Fals-Stewart, Kelley, Fincham, Golden et Logsdan, 2004). On note aussi des taux élevés de problèmes extériorisés (hyperactivité, troubles de comportement), de problèmes intériorisés (anxiété, dépression) (Arria, Mericle, Meyer et Winters, 2012; Fals-Stewart et al., 2004; Scaife, 2008; Vidal et al., 2012), des difficultés sur le plan cognitif, des retards scolaires (Vitaro, Assaad et Carbonneau, 2004) et un risque 
accru que les enfants développent eux-mêmes des problèmes de consommation (Conners et al., 2004; Dunn, Tarter, Mezzich, Vanyukov, Kirisci et Kirillova, 2002).

Au Québec, depuis 2008, deux programmes d'entrainement aux habiletés parentales (PEHP) destinés aux parents ayant une dépendance à l'alcool ou aux drogues et à leurs enfants de 6-12 ans ont été implantés dans certains centres de réadaptation en dépendance (CRD) : le programme 6-12 (CRDM-IU, 2012) et le Programme d'Intervention Appui aux Familles [PIAF, adaptation française du Strengthening Family Program (Kumpfer, 1997) ${ }^{1}$. Ainsi, ces programmes psychoéducatifs ont pour principaux objectifs d'accroitre les facteurs de protection (améliorer les relations familiales, augmenter les compétences parentales et les compétences sociales des enfants) et de diminuer les facteurs de risque (diminuer les problèmes de comportement des enfants) chez les familles ciblées. De façon plus spécifique, ces programmes visent à permettre au parent ayant une dépendance et à leur enfant âgé de 6 à 12 ans (1) de mieux comprendre les impacts de la dépendance du parent sur l'enfant et sur son rôle de parent; (2) de réduire les répercussions de la dépendance parentale sur la vie familiale et (3) d'expérimenter avec l'enfant et le parent différents outils destinés à mieux se connaitre et à mieux communiquer au sein de la famille (Laventure, Charbonneau, Sarrazin et Bertrand, 2013).

Les programmes se composent en moyenne de 12 à 15 rencontres de groupes de familles. Les rencontres, d'une durée de trois heures, ont lieu chaque semaine et prévoient des moments de convivialité familiale. La première heure est consacrée à un repas pris en groupe. Le repas communautaire initial permet de créer une dynamique positive offrant ainsi une fenêtre d'observation des dynamiques familiales dans une ambiance naturelle et selon un format d'accompagnement. Le repas est suivi d'une intervention simultanée d'une heure auprès des parents et des enfants. Après la première heure d'activité, tous se réunissent pour une activité familiale afin de mettre en œuvre les acquis entre les parents et les enfants et de faire un retour sur leurs activités respectives. Différents thèmes concernant les impacts de la consommation d'alcool et de drogues des parents sont alors abordés en toile de fond: la communication, la résolution de problèmes, les limites, les émotions, la gestion de la colère. Tous les services rattachés à ce programme sont gratuits et garantissent la confidentialité. Par ailleurs, des séances supplémentaires de soutien individualisé sont prévues pour maintenir et consolider les acquis du programme.

Pour bien évaluer l'implantation de tels programmes, l'Organisation mondiale de la santé en collaboration avec le United Nations International Drug Control Program et l'European Monitoring Centre for Drugs and Drug Addiction a établi des lignes directrices (Marsden, Ogbourne, Farrell et Rush, 2000). Basée sur ces lignes directrices et telle que recommandé par Marsden et coll. (2000), l'évaluation de l'implantation doit, entre autres aspects, permettre de décrire les

1. L'adaptation québécoise du programme a principalement consisté en sa traduction et en l'ajustement de l'atelier portant sur la dépendance qui n'était pas adapté aux approches d'intervention québécoises en dépendance (abstinence totale c. réduction des méfaits). 
caractéristiques des clients participant au programme. En effet, dans un processus d'implantation, il faut s'assurer que les familles participant au programme évalué correspondent aux familles ciblées par ledit programme (Rossi, Lipsey et Freeman, 2003). Connaitre les caractéristiques des familles rejointes par ces programmes permet ainsi de s'assurer que les objectifs du programme correspondent aux besoins des familles ciblées. Cette adéquation entre les objectifs du programme et les caractéristiques des familles rejointes est d'autant plus importante qu'elle est associée à une meilleure rétention de la clientèle dans le programme et ultimement à son efficacité. Dans cette optique, la présente étude vise à identifier les caractéristiques des familles qui ont participé à un PEHP-Dépendance en CRD au Québec et vérifier si ces familles représentent un groupe homogène.

\section{Méthode}

La présente étude est basée sur un devis transversal, exploratoire et descriptif. Les analyses présentées s'inscrivent dans un processus d'évaluation d'implantation des PEHP développé spécifiquement pour les parents ayant une dépendance à l'alcool et/ou aux drogues (Laventure et coll., 2013). Bien que cinq CRD aient implanté le PEHP-Dépendance au cours des dernières années, les données utilisées dans la présente étude ont été recueillies auprès de la clientèle d'un seul d'entre eux, le site ayant accueilli le plus grand nombre de familles parmi tous les sites. Ce choix a permis une standardisation des évaluations puisqu'elles ont été réalisées par un nombre restreint d'intervenants. Ce projet a été approuvé par le Comité d'éthique de la recherche en dépendance.

\section{Critères de sélection}

Pour participer au PEHP-Dépendance, la famille devait être composée d'au moins un parent ayant une dépendance à l'alcool ou aux drogues et avoir au moins un enfant âgé entre 6 et 12 ans présentant un problème intériorisé ou extériorisé. Pour venir aux rencontres, les enfants devaient être accompagnés du parent présentant un problème de dépendance ou encore d'un adulte significatif. Les enfants placés en famille d'accueil pouvaient également participer au programme en compagnie de leur parent.

\section{Participants}

L'échantillon est composé de 80 familles et de 108 enfants de 6 à 12 ans ayant participé au PEHP-Dépendance entre 2010 et 2014. Les répondants aux questionnaires d'évaluation sont les mères (84,4 \%) ou les pères (15,6\%). Ils ont 38,9 ans en moyenne (é.t. $=7,33$ ). Chez les enfants, $48,1 \%$ sont des garçons âgés en moyenne de 8,8 ans (é.t. $=1,75)$ et les filles $(52,9 \%$ ) âgées en moyenne 8,7 ans (é.t. = 1,95).

\section{Déroulement de l'étude}

Le projet s'inscrivant dans l'évaluation d'implantation des PEHPDépendance en CRD, les intervenants impliqués dans ce programme avaient pour tâche d'identifier les familles pouvant bénéficier d'un tel programme parmi 
les usagers du CRD. Après avoir accepté de participer au PEHP-Dépendance, les familles étaient invitées par les intervenants du PEHP à prendre part au projet de recherche. Le cas échéant, le formulaire de consentement était signé, puis les questionnaires d'évaluation remplis par le parent avant d'être transmis à l'équipe de recherche. Les questionnaires ont été remplis avant que les rencontres de groupe ne soient amorcées et ils constituaient une composante déjà prévue dans l'implantation du PEHP-Dépendance. Tous les questionnaires ont été remplis uniquement par le parent dépendant. Lorsque le parent avait plus d'un enfant qui participait au PEHP-Dépendance, il devait remplir un questionnaire par enfant.

\section{Instruments}

Le portrait clinique des familles a été documenté à l'aide de questionnaires inclus dans le Protocole d'Évaluation Multidimensionnel pour Enfants (PEME) qui a été développé spécifiquement pour les parents d'enfants de 6-12 ans par le Groupe de Recherche sur les Inadaptations Sociales de l'Enfance (GRISE, 2003).

Données socio-économiques. Le questionnaire utilisé pour évaluer cet aspect est inspiré de celui utilisé dans l'Enquête sur la santé mentale des jeunes de 6-14 ans (Breton, Valla et Bergeron, 1992). II permet de documenter différentes variables liées à la composition de la maisonnée (type de famille, placement en milieu substitut) et le profil sociodémographique des familles (scolarité, occupation).

État de santé psychologique des parents. L'état psychologique du parent a été évalué à l'aide de l'Indice de Détresse Psychologique de l'Enquête Santé Québec (IDPESQ-29; Préville, Boyer, Potvin, Perrault et Légaré, 1992) qui contient 29 items dont la réponse est évaluée sur une échelle de type Likert variant de 1 " Jamais » à 4 « Très souvent ». Il permet d'évaluer l'état de santé psychologique du répondant au cours des sept jours précédant l'évaluation sur quatre dimensions : les symptômes d'anxiété, les symptômes dépressifs, les symptômes d'irritabilité et les problèmes cognitifs. Les qualités psychométriques de cet outil sont satisfaisantes, les indices de cohérence interne variant de 0,77 à 0,85 pour chacune des souséchelles (Préville et coll., 1992). Un score global peut être calculé pour estimer le sentiment global de détresse psychologique. Un seuil clinique fixé au 80 e percentile de la distribution des valeurs de l'enquête Santé Québec est retenu comme seuil indiquant la présence de symptômes significatifs (Boyer, Préville, Légaré et Valois, 1993)

Problèmes de comportement de l'enfant. La version française du Child Behavior Checklist (Achenbach, 1991) a permis l'évaluation du portrait comportemental des jeunes âgés de 6 à 12 ans au cours des six derniers mois. II comporte 115 items de type Likert en trois points allant de 0 "Ne s'applique pas » à 2 "Toujours ou souvent vrai ». II évalue plusieurs échelles comportementales reliées au DSM-IV (APA, 1994). Dans le présent projet, les échelles retenues sont : comportements délinquants, comportements agressifs, anxiété, retrait/dépression, somatisation, problèmes sociaux, problèmes d'attention et trouble d'opposition. Les qualités psychométriques de la version originale du Child Behavior Checklist sont très bonnes (Achenbach et Rescorla, 2001). Les indices de cohérence interne varient entre 0,72 et 0,97 selon les échelles et les coefficients de fidélité test-retest 


\section{8}

varient entre 0,60 et 0,96 . Les qualités psychométriques de la version française sont tout aussi bonnes, la cohérence interne variant entre 0,57 à 0,88 (GRISE, 2003). Des scores globaux sont utilisés pour déterminer la présence de problèmes intériorisés et extériorisés. Établis par les auteurs du questionnaire, des seuils cliniques correspondants au sexe et à l'âge des enfants permettent d'établir la présence d'un problème chez le jeune pour chacune des échelles.

Stress familiaux. Une adaptation du Family Inventory of Life Events and Changes (McCubbin et Patterson, 1983) a été utilisée pour évaluer les évènements normatifs et non normatifs de la vie familiale de même que les transitions et les contraintes que peut connaitre une famille au cours de la dernière année. Ce questionnaire permet d'identifier les évènements vécus et le niveau de stress associé à ceux-ci à l'aide d'une liste de 28 évènements différents estimés sur une échelle de type Likert en cinq points variant de 1 «Aucunement stressant » à 5 «Extrêmement stressant ». Seuls le nombre et la nature de stresseurs différents vécus par les familles au cours de la dernière année ont été utilisés.

Pratiques éducatives. Ce questionnaire, version française de l'Alabama Parenting Questionnaire (Frick, 1991), permet d'identifier les pratiques éducatives du parent à l'égard de son enfant. Composé de 42 items de type Likert en cinq points allant de 1 " Jamais » à 5 " Toujours », ce questionnaire mesure cinq dimensions dont quatre ont été retenues pour la présente étude, deux échelles positives et deux échelles négatives. L'engagement parental qui se définit par la responsabilité du parent pour le bien-être général de l'enfant, l'accessibilité et la disponibilité du parent (Pleck, 2010), les comportements éducatifs positifs qui se définissent par l'utilisation de renforcements pour souligner les comportements positifs de l'enfant, le manque de supervision parentale qui se définit par le faible ou l'absence d'encadrement offert par la présence du parent, sa participation ou le suivi des activités de son enfant (Hamel, 2001) et la discipline inconstante qui est définit par l'utilisation inconstante de moyens, tant coercitifs que positifs employés par le parent pour contrôler le comportement de son enfant (Hamel, 2001). Certains items évaluant l'utilisation d'autres types de pratiques éducatives (dont l'utilisation de la punition) étaient également présents dans le questionnaire, mais ils ne sont pas retenus pour la présente étude considérant les faibles qualités psychométriques de cette échelle. Les qualités psychométriques de la version originale de cet outil sont satisfaisantes, les coefficients de cohérence interne varient entre 0,67 et 0,80 et les coefficients test-retest varient entre 0,66 et 0,89 selon les échelles (Shelton, Frick et Wooton, 1996). Des analyses préliminaires des versions françaises ont été réalisées auprès de deux populations cliniques, la cohérence interne, excluant l'échelle de punition corporelle, varie de 0,57 à 0,74 (Toupin et Pauzé, 2003). Un seuil clinique indicatif d'un problème aux différentes échelles a été fixé à deux écarts-types au-dessus de la moyenne. En ce sens, pour les échelles positives, un score total inférieur à 3,25 donne un indice que le parent fait preuve de pratiques éducatives positives. Alors que pour les échelles négatives, un score total supérieur à 2,5 donne un indice que le parent fait preuve de pratiques éducatives négatives.

Fonctionnement familial. Ce questionnaire, version française du Family Assessment Device (Epstein, Baldwin et Bishop, 1983) évalue différents aspects du fonctionnement de la famille. Initialement composée de 60 items, la version utilisée 
pour le projet comporte 45 items, les items de deux échelles moins valides auprès de certains échantillons cliniques ayant été retirés. Chaque item doit être répondu à l'aide d'une échelle de type Likert en 4 points variant de 1 "Fortement en accord » à 4 «Fortement en désaccord » et permet d'évaluer la capacité de résolution de problèmes familiaux (6 items), la communication familiale (9 items), le partage des rôles familiaux (11 items) et l'investissement affectif dans la famille (7 items). Une sous-échelle de fonctionnement général compose également le questionnaire (12 items). La cohérence interne est satisfaisante les alphas de Cronbach variant de 0,68 à 0,87 (Epstein et coll., 1983). Des analyses préliminaires des versions françaises ont été réalisées auprès de différentes populations cliniques afin de vérifier la cohérence interne et la fidélité test-retest de chaque sous-échelle. En général, les premiers résultats sont bons et permettent de croire que la version française de l'outil a conservé ses qualités psychométriques (Pauzé, 2007). Un seuil clinique indicatif d'un problème aux différentes échelles a été fixé à deux écarts-types audessus de la moyenne. En ce sens, pour les échelles de résolution de problèmes et de communication, un score total inférieur à 2,2 donne un indice que la famille éprouve des difficultés sur ces aspects. Alors qu'un score inférieur à 2,3 à l'échelle des rôles, que 2,1 à l'échelle d'investissement affectif et que 2,0 au fonctionnement général indique que la famille éprouve des difficultés sur ces aspects.

Disponibilité des ressources. Ce questionnaire est la traduction de l'échelle Perceived Adequacy of Resources Scale (Rowland, Richard et Sharon, 1985). Elle vise à mesurer la perception qu'a l'individu quant aux ressources dont il dispose. Quatre échelles de la version française ont été utilisées pour l'étude (ressources liées à l'environnement physique, ressources temporelles, ressources financières, ressources interpersonnelles). Celles-ci sont composées de quatre items chacune et elles sont estimées sur une échelle de type Likert en quatre points allant de 1 "Fortement en désaccord " à 4 "Fortement en accord ». Rowland et coll. (1985) indiquent une bonne cohérence interne de l'instrument (alpha de Cronbach globale $=0,89$ ), tout comme l'évaluation des qualités psychométriques de la version française de l'instrument réalisée par le GRISE (2003) (alphas de Cronbach pour chacune des échelles variant de 0,73 à 0,91$)$. Un seuil clinique indicatif d'un problème à l'échelle a été fixé à deux écarts-types au-dessus de la moyenne. En ce sens, pour l'échelle de l'environnement physique le seuil clinique se situe à 2,98, pour les ressources temporelles, le seuil clinique est de 2,25, pour les ressources financières, il est de 2,01 et pour les ressources interpersonnelles de 2,89 . Un score plus faible que les scores établis signifie que la famille présente certains manques au niveau de la ressource.

\section{Analyses}

Dans un premier temps, des analyses descriptives (moyennes et fréquences) ont été utilisées pour décrire les familles et les enfants qui participent à un PEHPDépendance. Puis, pour dresser les profils types des familles participantes au PEHP-Dépendance, différentes analyses ont été réalisées. D’abord, afin de garder l'indépendance des observations dans l'analyse de classification, un seul enfant par famille a été sélectionné aléatoirement afin que chaque famille ne soit représentée qu'une seule fois dans l'échantillon. Ceci a permis d'obtenir 80 familles et 80 enfants plutôt que 80 familles et 108 enfants. Par la suite une analyse en composante 
principale a été effectuée afin de diminuer le nombre de variables pouvant être utilisées pour dresser le profil des familles, car celui-ci était trop important pour procéder à une classification hiérarchique interprétable. Cette analyse a permis d'identifier cinq valeurs propres supérieures à 1 dont deux représentaient $52 \%$ de la variance expliquée; les trois autres représentant respectivement 9,2 \%, 6,8 \% et $5,8 \%$ de la variance expliquée. Considérant l'importance des deux premiers facteurs (état de santé psychologique du parent et du fonctionnement familial) de même que leur apport clinique, seuls ceux-ci ont été conservés pour l'analyse subséquente. Par la suite, une analyse de classification hiérarchique par la méthode d'agrégation a été effectuée en utilisant les 75 familles pour lesquelles tous les questionnaires étaient remplis; les cinq familles retirées de l'échantillon ayant toutes au moins deux questionnaires non remplis.

\section{Résultats}

Cette section est divisée en deux parties. La première présente les caractéristiques des familles selon les sphères évaluées. La seconde partie présente les quatre profils-types des familles qui participent à un PEHP-Dépendance.

\section{Portrait des familles}

\section{Caractéristiques socio-économiques}

Au moment de compléter l'évaluation, la majorité des enfants vivaient dans une famille monoparentale maternelle $(43,5 \%)$ ou dans une famille intacte (dont les deux parents vivent ensemble) (30,6 \%). De plus, 17,6 \% des enfants participant au PEHP-Dépendance avaient déjà été placés, au moins à une reprise, en milieu substitut au cours de leur vie.

La majorité des parents avaient un diplôme d'études secondaires ou professionnelles $(55,4 \%)$, près de la moitié des parents $(45,9 \%)$ recevaient de l'aide sociale et la majorité des parents (44,3\%) avaient un revenu familial annuel de moins de 20000 \$. Le tableau 1 présente l'ensemble des caractéristiques socioéconomiques des familles ayant participé au PEHP-Dépendance.

\section{État de santé psychologique des parents}

Outre la présence de dépendance à l'alcool et aux drogues, les problèmes de santé psychologique présents chez les parents de l'échantillon étaient, en ordre décroissant, la dépression (41,9\%), la détresse psychologique (40,3\%), l'irritabilité (37\%), l'anxiété $(36 \%)$ et les problèmes cognitifs $(12 \%)$. Près de la moitié des parents ne présentaient aucun problème psychologique rencontrant le seuil clinique $(46,3 \%)$ et $10,0 \%$ rencontraient le seuil clinique pour les cinq problèmes psychologiques évalués. 
Tableau 1. Conditions socio-économiques des familles

\begin{tabular}{|c|c|c|c|}
\hline \multicolumn{2}{|l|}{ Structure familiale $(N=80)$} & \multicolumn{2}{|c|}{ Occupation principale du parent $(N=80)$} \\
\hline Monoparentale (mère) & $43,5 \%$ & Aide sociale & $45,9 \%$ \\
\hline Famille intacte & $30,6 \%$ & Travail à temps complet & $24,3 \%$ \\
\hline Famille accueil & $8,3 \%$ & Autres (retraite, invalidité, études) & $12,2 \%$ \\
\hline Monoparentale (père) & $6,5 \%$ & Travail à temps partiel & $9,5 \%$ \\
\hline Recomposée & $6,5 \%$ & Tenir maison & $8,1 \%$ \\
\hline Autre & $4,6 \%$ & & \\
\hline \multicolumn{2}{|c|}{ Niveau d'études des parents $(N=80)$} & \multicolumn{2}{|l|}{ Placement en milieu substitut } \\
\hline Aucun diplôme & $19,0 \%$ & $\begin{array}{l}\text { Du parent au cours de son } \\
\text { enfance }(N=80)\end{array}$ & $25,0 \%$ \\
\hline Diplôme d'études sec. ou prof. & $55,4 \%$ & De l'enfant $(N=106)$ & $17,6 \%$ \\
\hline Diplôme collégial & $14,9 \%$ & & \\
\hline Diplôme universitaire & $10,8 \%$ & & \\
\hline \multicolumn{2}{|l|}{ Revenu familial annuel $(N=80)$} & & \\
\hline Moins de $20000 \$$ & $44,3 \%$ & & \\
\hline Entre $20000 \$$ et $40000 \$$ & $32,7 \%$ & & \\
\hline $40000 \$$ et plus & $23,0 \%$ & & \\
\hline
\end{tabular}

\section{Caractéristiques personnelles des enfants}

Tel que rapporté par les parents, 50,9\% des enfants inclus dans le PEHPDépendance présentaient un trouble intériorisé ou un trouble extériorisé, $27,8 \%$ présentaient les deux troubles à la fois. Plus précisément, $37,7 \%$ présentaient des troubles intériorisés et $42,5 \%$ présentaient des troubles extériorisés. De plus, $19,8 \%$ des enfants présentaient des difficultés sans pour autant atteindre le seuil clinique d'un trouble intériorisé ou extériorisé.

Les difficultés les plus présentes chez les enfants de l'échantillon étaient les comportements agressifs $(25,5 \%)$, le trouble de l'opposition $(24,5 \%)$ et les comportements délinquants $(22,6 \%)$. Les problèmes les moins prévalents chez les enfants étaient les problèmes d'attention $(8,5 \%)$ et la somatisation $(6,6 \%)$. Le tableau 2 présente la prévalence de l'ensemble des problèmes de santé mentale évalués chez les enfants.

\section{Caractéristiques familiales}

Le tableau 3 présente les caractéristiques des familles.

Pratiques éducatives. Les deux pratiques éducatives les plus présentes chez les parents de l'échantillon étaient la discipline parentale inconstante (ex. cesser une punition plus tôt que mentionné) $(53,5 \%$ ) et le manque d'engagement 
Tableau 2. Prévalence des problèmes de santé mentale chez les enfants

\begin{tabular}{lc}
\hline Problèmes de santé mentale & Présence des problèmes $(N=106)$ \\
\hline Problèmes extériorisés & $42,5 \%$ \\
Problèmes intériorisés & $37,7 \%$ \\
Problèmes intériorisés ou problèmes extériorisés & $50,9 \%$ \\
Problèmes intériorisés et problèmes extériorisés & $27,8 \%$ \\
Comportements agressifs & $25,5 \%$ \\
Trouble d'opposition & $24,5 \%$ \\
Comportements délinquants & $22,6 \%$ \\
Retrait/Dépression & $18,9 \%$ \\
Anxiété & $18,9 \%$ \\
Problèmes sociaux & $14,2 \%$ \\
Problèmes d'attention & $8,5 \%$ \\
Somatisation & $6,6 \%$ \\
\hline
\end{tabular}

parental (manque d'intérêt à l'endroit de son enfant, de ses activités ou de ses fréquentations) envers son enfant $(22,4 \%)$. Fait à souligner, près de la moitié des parents $(44,4 \%)$ ne présentaient aucune pratique éducative ayant des lacunes suffisamment grandes pour rencontrer le seuil clinique et aucun parent ne rencontrait le seuil clinique pour l'ensemble des pratiques éducatives évaluées.

Fonctionnement parental. Les problèmes de fonctionnement familial les plus présents dans les familles participant au PEHP-Dépendance étaient la présence d'un faible partage des rôles familiaux (déséquilibre quant à la distribution des responsabilités familiales, parentification) $(61,3 \%)$ et un faible investissement affectif $(45,0 \%)$. Ici, encore, $26,3 \%$ des familles ne présentaient aucune difficulté liée au fonctionnement familial et $10,0 \%$ des familles rencontraient le seuil clinique pour l'ensemble des composantes du fonctionnement familial évaluées.

Ressources des familles. La moitié des familles manquaient de ressources financières $(50,0 \%$ ) et de ressources interpersonnelles (membres de la famille ou amis sur qui ils peuvent compter) (49,3\%) au moment de leur entrée au PEHP-Dépendance. Peu de familles présentaient des lacunes sur l'ensemble des quatre types de ressources évaluées $(5,1 \%)$, mais plusieurs présentaient des lacunes importantes pour un ou deux $(60,3 \%)$ types de ressources.

Stress familiaux. En ce qui concerne les stress familiaux vécus dans la dernière année, les familles inscrites au PEHP-Dépendance avaient vécu en moyenne six des 28 stresseurs évalués $(M=6,39$, é.t. $=4,31)$ au cours de l'année précédant leur entrée au PEHP. Les plus fréquemment vécus au cours de la dernière année étaient : la présence de conflits importants entre les parents concernant les enfants $(46,3 \%)$, une surcharge de travail $(32,5 \%)$, un membre de la famille a subi une agression sexuelle (28,8 \%), un enfant a fait une fugue de la maison $(23,8 \%)$ et un des parents est peu présent à la maison $(23,8 \%)$. 


\begin{tabular}{|c|c|c|c|}
\hline \multirow[b]{2}{*}{ Pratiques éducatives } & \multirow{2}{*}{$\begin{array}{c}\begin{array}{c}\text { Présence } \\
\text { du seuil } \\
\text { clinique }\end{array} \\
\%\end{array}$} & \multicolumn{2}{|c|}{$\begin{array}{l}\text { Nombre de catégories } \\
\text { rencontrant le seuil } \\
\text { clinique }\end{array}$} \\
\hline & & Nombre & $\%$ \\
\hline Discipline parentale inconstante $(N=99)$ & $53,5 \%$ & 0 & $44,4 \%$ \\
\hline Manque d'engagement parental $(N=98)$ & $22,4 \%$ & 1 & $33,3 \%$ \\
\hline Manque de pratiques éducatives positives $(N=103)$ & $7,8 \%$ & 4 & $0 \%$ \\
\hline Manque de supervision parentale $(N=100)$ & $3,0 \%$ & $2-3$ & $21,3 \%$ \\
\hline \multicolumn{4}{|l|}{ Fonctionnement familial } \\
\hline Faible partage des rôles familiaux $(N=75)$ & $61,3 \%$ & 0 & $26,3 \%$ \\
\hline Faible investissement affectif dans la famille $(N=75)$ & $45,0 \%$ & $1-2$ & $32,6 \%$ \\
\hline Manque de communication familiale $(N=76)$ & $33,8 \%$ & 5 & $10,0 \%$ \\
\hline Faible résolution de problèmes familiaux $(N=75)$ & $32,5 \%$ & $3-4$ & $28,8 \%$ \\
\hline Dysfonctionnement familial général $(N=71)$ & $28,8 \%$ & & \\
\hline \multicolumn{4}{|l|}{ Ressources des familles } \\
\hline $\begin{array}{l}\text { Manque de ressources liées à l'environnement } \\
\text { physique }(N=73)\end{array}$ & $32,9 \%$ & 0 & $17,9 \%$ \\
\hline Manque de ressources financières $(N=74)$ & $50,0 \%$ & 3 & $16,7 \%$ \\
\hline Manque de ressources interpersonnelles $(N=75)$ & $49,3 \%$ & 4 & $5,1 \%$ \\
\hline Manque de ressources temporelles $(N=72)$ & $23,6 \%$ & $1-2$ & $60,3 \%$ \\
\hline
\end{tabular}

\section{Profils-types des familles participant à un PEHP-Dépendance}

Les analyses de classification hiérarchique ont révélé quatre profilstypes de famille différents parmi l'ensemble des familles s'étant inscrites et PEHP-Dépendance: les familles ayant peu de difficultés de fonctionnement ou psychologiques (regroupant 34 familles/75), les familles ayant des difficultés de fonctionnement familial (regroupant 12 familles/75), les familles dont les parents ont des difficultés psychologiques (regroupant 12 familles/75) et les familles ayant à la fois des difficultés de fonctionnement familial et dont les parents ont des difficultés psychologiques (regroupant 17 familles/75). Le tableau 4 présente l'ensemble des caractéristiques des familles et des enfants selon les quatre profils de famille. 
Tableau 4. Caractéristiques des familles et des enfants selon leur profil type

\begin{tabular}{|c|c|c|c|c|}
\hline & $\begin{array}{r}\text { Profil } \\
\text { type } 1\end{array}$ & $\begin{array}{c}\text { Profil } \\
\text { type } 2\end{array}$ & $\begin{array}{l}\text { Profil } \\
\text { type } 3 \\
\end{array}$ & $\begin{array}{l}\text { Profil } \\
\text { type } 4\end{array}$ \\
\hline \multicolumn{5}{|l|}{ Type de famille } \\
\hline Familles intactes & $38,2 \%$ & $25,0 \%$ & $8,3 \%$ & $23,5 \%$ \\
\hline Monoparentales/Recomposées & $50,0 \%$ & $58,3 \%$ & $83,3 \%$ & $58,8 \%$ \\
\hline Famille d'accueil & $5,9 \%$ & $16,7 \%$ & $0 \%$ & $5,9 \%$ \\
\hline Autre & $5,9 \%$ & $0 \%$ & $8,3 \%$ & $11,8 \%$ \\
\hline \multicolumn{5}{|l|}{ Scolarité } \\
\hline Au moins 1 année primaire complété & $0 \%$ & $9,1 \%$ & $0 \%$ & $6,3 \%$ \\
\hline Au moins 1 année secondaire complétée & $51,5 \%$ & $63,6 \%$ & $60,0 \%$ & $62,5 \%$ \\
\hline Diplôme d'études professionnelles obtenu & $12,1 \%$ & $9,1 \%$ & $20,0 \%$ & $12,5 \%$ \\
\hline Diplôme d'études collégiales obtenu & $18,2 \%$ & $18,2 \%$ & $20,0 \%$ & $6,3 \%$ \\
\hline Diplôme universitaire obtenu & $18,2 \%$ & $0 \%$ & $0 \%$ & $12,5 \%$ \\
\hline \multicolumn{5}{|l|}{ Occupation } \\
\hline Travail temps plein & $27,3 \%$ & $9,1 \%$ & $40,0 \%$ & $25,0 \%$ \\
\hline Travail temps partiel & $18,2 \%$ & $9,1 \%$ & $0 \%$ & $6,3 \%$ \\
\hline Aide social & $36,4 \%$ & $53,6 \%$ & $40,0 \%$ & $50,0 \%$ \\
\hline Chômage & $3,0 \%$ & $0 \%$ & $10,0 \%$ & $6,3 \%$ \\
\hline Congé maternité/maladie & $3,0 \%$ & $0 \%$ & $0 \%$ & $12,6 \%$ \\
\hline Autre & $12,1 \%$ & $18,2 \%$ & $10,0 \%$ & $0 \%$ \\
\hline \multicolumn{5}{|l|}{ Revenu familial annuel } \\
\hline 0 à $19999 \$$ & $41,4 \%$ & $62,5 \%$ & $50,0 \%$ & $46,2 \%$ \\
\hline 20000 à $39999 \$$ & $27,6 \%$ & $25,0 \%$ & $25,0 \%$ & $38,5 \%$ \\
\hline 40000 et plus & $31,0 \%$ & $12,5 \%$ & $25,0 \%$ & $15,4 \%$ \\
\hline \multicolumn{5}{|l|}{ Placement } \\
\hline Du parent & $14,7 \%$ & $8,3 \%$ & $41,7 \%$ & $41,2 \%$ \\
\hline De l'enfant & $17,6 \%$ & $25,0 \%$ & $0 \%$ & $23,5 \%$ \\
\hline \multicolumn{5}{|l|}{ Santé mentale des enfants } \\
\hline Troubles intériorisés & $26,5 \%$ & $41,7 \%$ & $33,0 \%$ & $82,4 \%$ \\
\hline Troubles extériorisés & $29,4 \%$ & $50,0 \%$ & $33,0 \%$ & $82,4 \%$ \\
\hline Troubles intériorisés ou extériorisés & $26,9 \%$ & $50,0 \%$ & $41,7 \%$ & $88,2 \%$ \\
\hline Troubles intériorisés et extériorisés & $11,9 \%$ & $41,7 \%$ & $25,0 \%$ & $76,5 \%$ \\
\hline \multicolumn{5}{|l|}{ Fonctionnement familial } \\
\hline Faible partage des rôles familiaux & $33,3 \%$ & 100 & $5,0 \% 0$ & $100 \%$ \\
\hline Faible investissement affectif & $20,6 \%$ & $91,7 \%$ & $33,3 \%$ & $80,0 \%$ \\
\hline Manque de communication familiale & $6,1 \%$ & $50,0 \%$ & $16,7 \%$ & $93,8 \%$ \\
\hline Faible résolution de problèmes familiaux & $3,0 \%$ & $63,6 \%$ & $8,3 \%$ & $82,4 \%$ \\
\hline Dysfonctionnement familial général & $3,2 \%$ & $63,6 \%$ & $8,3 \%$ & $87,5 \%$ \\
\hline \multicolumn{5}{|l|}{ Pratiques éducatives } \\
\hline Discipline parentale inconstante & $48,4 \%$ & $58,3 \%$ & $66,7 \%$ & $76,5 \%$ \\
\hline Manque d'engagement parental & $9,1 \%$ & $9,1 \%$ & $8,3 \%$ & $56,3 \%$ \\
\hline Manque de pratiques éducatives positives & $8,8 \%$ & $0 \%$ & $0 \%$ & $17,6 \%$ \\
\hline Manque de supervision parentale & $3,0 \%$ & $8,3 \%$ & $0 \%$ & $5,9 \%$ \\
\hline \multicolumn{5}{|l|}{ Santé psychologique des parents } \\
\hline Détresse psychologique significative & $0 \%$ & $0 \%$ & $100 \%$ & $94,1 \%$ \\
\hline Symptômes significatifs de dépression & $5,9 \%$ & $16,7 \%$ & $91,7 \%$ & $94,1 \%$ \\
\hline Symptômes significatifs d'anxiété & $5,9 \%$ & $8,3 \%$ & $58,3 \%$ & $88,2 \%$ \\
\hline Symptômes significatifs d'irritabilité & $3,0 \%$ & $8,3 \%$ & $66,7 \%$ & $88,2 \%$ \\
\hline Problèmes cognitifs significatifs & $0 \%$ & $0 \%$ & $16,7 \%$ & $41,2 \%$ \\
\hline
\end{tabular}




\section{Profil type 1. Les familles ayant peu de difficultés de fonctionnement familial et psychologiques}

Les familles de ce profil type présentaient peu de difficultés tant en ce qui concerne la santé psychologique des parents que le fonctionnement familial. La moitié des familles présentant ce profil étaient monoparentales ou recomposées $(88,2 \%$ ). Les parents avaient en moyenne 40,1 ans (é.t. $=7,5)$ et $27,3 \%$ occupaient un travail rémunéré à temps complet alors que $36,4 \%$ vivaient de l'aide sociale. Le revenu familial annuel moyen de $41,4 \%$ de ces familles était inférieur à 19 999 \$ et $51,5 \%$ des parents de ce profil n'avaient pas obtenu de diplôme d'études secondaires. Les enfants de ces familles avaient en moyenne 8,4 ans (é.t. $=1,9$ ) et elles regroupaient plus de filles (58,8 \%) que de garçons $(41,2 \%) ; 14,7 \%$ des parents avaient vécu un placement à l'enfance et que $17,6 \%$ des enfants de ces familles vivaient présentement ou avaient également vécu un placement. Parmi les enfants de ce profil, $26,9 \%$ présentaient un trouble intériorisé ou un trouble extériorisé alors que $11,8 \%$ présentaient simultanément ces deux types de trouble.

Bien que les parents présentaient peu de difficultés psychologiques, les principales difficultés rencontrées sur ce plan étaient des symptômes significatifs de dépression (5,9\%) et ceux d'anxiété (5,9\%). Sur le plan des difficultés liées au fonctionnement familial, celles rencontrées par le plus grand nombre de familles de ce profil étaient celles liées au partage des rôles familiaux $(33,3 \%)$ et au faible investissement affectif (20,6\%). Sur le plan des pratiques éducatives, la discipline parentale inconstante $(48,4 \%)$ était la plus prévalente.

\section{Profil type 2. Les familles ayant des difficultés de fonctionnement familial}

Les familles de ce profil type se distinguaient principalement par la présence de difficultés liées au fonctionnement familial. La majorité des familles de ce type de profil étaient des familles monoparentales/recomposées $(58,3 \%)$. Les parents avaient en moyenne 40,0 ans (é.t. $=6,6)$ et la majorité $(63,6 \%)$ vivait de l'aide sociale. Le revenu familial annuel moyen de $62,5 \%$ de ces familles était inférieur à 19999 et 63,6\% des parents de ce profil n'avaient pas obtenu de diplôme d'études secondaires. Notons que parmi les quatre profils de familles répertoriés, celui-ci était le seul à présenter plus de garçons $(66,7 \%)$ que de filles $(33,3 \%)$. Les enfants avaient en moyenne 9,0 ans (é.t. $=1,4$ ) et $8,3 \%$ des parents avaient vécu un placement pendant l'enfance alors que $25,0 \%$ des enfants avaient vécu ou vivaient actuellement un placement. Parmi les enfants de ce profil, $50 \%$ présentaient un trouble intériorisé ou un trouble extériorisé et $41,7 \%$ présentaient ces deux types de troubles.

Dans ce type de famille, les difficultés psychologiques chez les parents étaient peu fréquente, la plus prévalente étant la dépression (16,7\%). Inversement, les difficultés de fonctionnement familial étaient nombreuses; toutes les difficultés de fonctionnement familial évaluées étaient présentes chez $50 \%$ et plus des familles. Les deux difficultés les plus présentes étaient celles reliées au partage des rôles familiaux (100 \%) et les problèmes d'investissement affectif (91,7\%). Par contre, ces familles présentaient peu de difficultés reliées aux pratiques éducatives. 
Seule la présence d'une discipline parentale inconstante était notée chez plus de la moitié des familles de ce profil (58,3\%).

\section{Profil type 3. Les familles dont les parents ont des difficultés psychologiques}

Les familles de ce profil type étaient celles qui se distinguaient par la présence d'importantes difficultés psychologiques chez les parents. Ce troisième type de famille se composait principalement de familles monoparentales ou recomposées (83,3\%). Les parents étaient âgés en moyenne de 34,6 ans (é.t. = 6,2 ) et $40,0 \%$ occupaient un travail rémunéré à temps plein alors que $40,0 \%$ recevaient de l'aide sociale. Le revenu familial annuel moyen de 50,0\% de ces familles était inférieur à 19999 \$ et 60,0 \% des parents des familles de ce profil n'avaient pas obtenu leur diplôme d'études secondaires. Ces familles regroupaient un peu plus de filles (58,3 \%) que de garçons (41,7 \%) et l'âge moyen des enfants était 8,8 ans (é.t.=1,9). Si les parents de ces familles étaient nombreux à avoir vécu un placement au cours de leur enfance $(41,7 \%)$, aucun de leurs enfants n'avait été placé. Parmi les enfants de ce profil, 41,7\% présentaient un trouble intériorisé ou un trouble extériorisé alors que $25,0 \%$ présentaient simultanément ces deux types de trouble.

Comme son nom l'indique, les parents de ce profil étaient nombreux à présenter des symptômes de problèmes de santé mentale. L'ensemble de parents présentait une détresse psychologique cliniquement significative et $91,7 \%$ d'entre eux présentaient d'importants symptômes dépressifs. Parmi les difficultés de fonctionnement familial rencontrées chez les familles de ce profil, les plus présentes étaient le faible partage des rôles familiaux $(50,0 \%)$ et le faible investissement affectif $(33,3 \%)$. Sur le plan des pratiques éducatives, la discipline parentale inconstante caractérisait davantage les familles de ce profil $(66,7 \%)$.

\section{Profil type 4. Les familles ayant à la fois des difficultés de fonctionnement familial et dont les parents ont des difficultés psychologiques}

Les familles de ce profil type étaient celles qui se distinguaient par la présence d'importantes difficultés, et ce, tant sur le plan des difficultés psychologiques chez les parents que par des difficultés liées au fonctionnement familial. Plus de la moitié des familles de ce profil type étaient monoparentales ou recomposées $(58,8 \%)$. Les parents étaient âgés en moyenne de 37,7 ans (é.t. $=6,5$ ) et $25,0 \%$ de ces parents occupaient un emploi rémunéré à temps plein. Le revenu familial annuel moyen de 46,2 \% de ces familles était inférieur à $19999 \$$ et $62,5 \%$ des parents de ces familles n'avaient pas obtenu un diplôme d'études secondaires. Ces familles comptaient légèrement plus de filles $(58,8 \%)$ que de garçons $(41,2 \%)$ et les enfants étaient âgés en moyenne de 8,5 ans (é.t. $=2,1)$. Près de la moitié des parents de ces familles avaient vécu un placement à l'enfance $(41,2 \%)$ et $23,5 \%$ des enfants de ces familles vivaient actuellement ou avaient vécu un placement. Parmi les enfants de ce profil, $88,2 \%$ présentaient un trouble intériorisé ou un trouble extériorisé et $76,5 \%$ présentaient ces deux types de trouble. 
Les difficultés psychologiques des parents étaient bien présentes chez les familles de ce profil puisque l'ensemble des difficultés évaluées se retrouvait chez plus de $40 \%$ des parents. Toutefois les plus présentes étaient la détresse psychologique $(94,1 \%)$ et la dépression $(94,1 \%)$. Sur le plan du fonctionnement familial, les cinq types de difficultés évalués étaient présents chez plus de $80 \%$ des familles. Les deux difficultés familiales les plus présentes étaient le faible partage des rôles familiaux (100 \%) et le manque de communication familiale $(93,8 \%)$. Des difficultés étaient également présentes sur le plan des pratiques éducatives et ici encore c'étaient les pratiques éducatives inconstantes (76,5\%) qu'on retrouvait chez le plus grand nombre de familles.

\section{Discussion}

La présente étude se situe dans le processus d'évaluation d'implantation des PEHP développés pour les parents ayant une dépendance à l'alcool ou aux drogues offerts dans certains CRD du Québec et avait pour objectifs d'identifier les caractéristiques des parents et des enfants participant aux PEHP-Dépendance offerts dans les CRD du Québec et de vérifier si ces familles formaient ou non un groupe homogène. Mieux connaitre les caractéristiques de ces familles permet de s'assurer que les PEHP-Dépendance rejoignent la clientèle pour laquelle ils ont été créés et d'assurer ainsi l'adéquation entre les besoins des familles et les objectifs de ces programmes.

Les résultats indiquaient d'abord que les familles inscrites aux PEHPDépendance sont des familles présentant un faible niveau socio-économique, une discipline parentale inconstante, un faible engagement parental, un fonctionnement familial déficient sur le plan du partage de rôles et de l'investissement affectif et un manque de ressources financières et interpersonnelles. Pour leur part, les enfants des familles étaient nombreux à présenter des troubles intériorisés et extériorisés. Soulignons d'abord que ces caractéristiques sont semblables à celles qui sont habituellement associées aux familles où la dépendance aux substances est présente (Barnard et McKeganey, 2004, Hogan et Higgins, 2001, Lussier et coll., 2010, Vitaro, Assaad et Carbonneau, 2004).

À la lumière des résultats obtenus, les familles inscrites au PEHPDépendance bénéficient à participer à ces programmes. Les différents ateliers des PEHP-Dépendance auxquels participaient les parents et les enfants ciblaient spécifiquement les difficultés présentées par les familles à l'étude. De plus, comme les PEHP-Dépendance étaient offerts en famille et en groupe, ils permettaient de favoriser l'engagement parental, l'investissement affectif et d'augmenter les ressources interpersonnelles des familles. En effet, en participant à un PEHPDépendance, ces familles voient l'opportunité d'avoir accès à des intervenants spécialisés et à côtoyer d'autres familles qui vivent les mêmes difficultés. En somme, l'ensemble des difficultés rencontrées par les familles participant aux PEHP-Dépendance, peut expliquer, du moins en partie, les raisons pour lesquelles ces parents s'inscrivent à un tel programme. Le fait que les PEHP-Dépendance soient créés spécifiquement et uniquement pour les familles où la dépendance à l'alcool et aux drogues est présente peut permettre d'offrir un milieu de soutien où 
les craintes de jugement et de stigmatisation sont moins présentes (Charbonneau, 2011; Klee, Jackson et Lewis, 2002).

Les résultats obtenus ont également permis d'identifier quatre profils-types distincts selon les difficultés familiales présentées et les difficultés psychologiques présentés par les parents. Le profil type de famille le plus représenté (45\%) est composé des familles avec peu de difficultés liées au fonctionnement familial et psychologique. Les trois autres profils-types de familles sont représentés dans des proportions comparables: les familles pour lesquelles les difficultés sont principalement liées au fonctionnement familial (16\%), les familles pour lesquelles les difficultés sont principalement liées aux difficultés psychologiques des parents (16\%) et les familles ayant à la fois des difficultés familiales et dont les parents ont des difficultés psychologiques (23\%).

II semble assez simple de comprendre que les familles qui présentent davantage de difficultés soient inscrites au PEHP-Dépendance. En effet, les parents qui rapportent et donc reconnaissent davantage de difficultés peuvent vivre plus d'inconfort par rapport à leur consommation d'alcool ou de drogues et, par le fait même, voient leur participation à un PEHP-Dépendance comme une occasion de recevoir de l'aide pour eux et leur enfant. II est toutefois plus difficile de comprendre les raisons pour lesquelles les parents rapportant moins de difficultés (profil type 1) se sont inscrits à un tel programme. Comme les parents n'ont pas été interrogés sur leur motivation à participer aux PEHP-Dépendance, seules des hypothèses peuvent être émises pour expliquer ce résultat. Soulignons que même si certains parents rapportent moins de difficultés familiales et personnelles que les autres parents à l'étude, ils ont tout de même une dépendance à l'alcool ou aux drogues et leurs enfants vivent des impacts de cette consommation. Par exemple, peut être se sentent-ils coupables de leur consommation et de l'impact de celle-ci sur leur famille et qu'ils désirent être un meilleur parent pour leurs enfants (Barnard, 2006; Venne et Morissette, 2009).

La présence de quatre profils- types distincts de famille questionne également la pertinence de regrouper des familles qui ont des difficultés différentes dans un même programme. Comme les facteurs thérapeutiques sont plus aisément activés par une forte cohésion dans une intervention de groupe (Crouch, Bloch et Wanlass, 1994; Yalom et Leszcz, 2005), l'impact de tel programme dans un contexte d'hétérogénéité des participants peut être moindre. Inversement, sur le plan clinique, l'hétérogénéité des familles participantes à un PEHP-Dépendance pourrait représenter une force. En effet, le fait de réunir des familles dont les parents présentent tous une dépendance peut permettre un soutien mutuel et permettre aux familles d'apprendre les unes des autres puisqu'elles ne présentent pas toutes les difficultés au même niveau (Sword, Jack, Niccols, Milligan, Henderson et Thabane, 2009). Ainsi, l'hétérogénéité des familles dans une intervention de groupe pourrait avoir des impacts négatifs en limitant l'engagement des parents (Koerting et al., 2013; Mytton, Ingram, Manns et Thomas, 2014) ou des impacts positifs en permettant un soutien mutuel (Sword et coll., 2009). De futures études comparant l'efficacité des PEHP selon des profils-types des familles pourraient permettre de répondre à ces questions et, éventuellement d'ajuster les critères de sélection des participants aux PEHP-Dépendance. 


\section{Conclusion}

Bien qu'il s'agit d'une première étape dans l'évaluation d'implantation et d'efficacité des PEHP-Dépendance offerts depuis quelques années au Québec, la présente étude a permis de mettre en lumière les caractéristiques des familles qui s'inscrivent à un PEHP-Dépendance dans un CRD et de démontrer la présence de quatre profils-types de familles parmi celles-ci. En connaissant mieux ces caractéristiques et ces profils-types, il sera possible d'adapter les PEHP-Dépendance afin qu'ils répondent davantage aux besoins de ces familles et optimiser les retombées positives pour celles-ci.

Les résultats de la présente étude doivent tout de même être nuancés par la présence de certaines limites. Une première limite réside dans le fait que le portrait des familles a été tiré des évaluations des familles participant aux PEHPDépendance et tient compte uniquement du point de vue du parent dépendant. Une évaluation multirépondants, incluant l'enfant, aurait pu limiter les biais de désirabilité sociale souvent présents chez les parents dépendants à l'alcool et aux drogues. Une seconde limite réside dans l'aspect de volontariat des familles quant à leur participation à la recherche. II est possible que les caractéristiques des familles réfractaires à la recherche se distinguent de celles des familles ayant accepté d'y participer et que les résultats obtenus reflètent le fait que les familles présentant des profils moins sévères sont plus nombreuses à avoir accepté de participer à la présente étude. Une troisième limite réside dans la présence importante des mères dans l'échantillon. En effet, bien que ceci soit représentatif des parents consommateurs participant dans des programmes d'intervention familiaux (Blanchette-Martin, Ferland, et Pelletier, 2015), cela rend difficile la généralisation des résultats à l'ensemble des parents dépendants à l'alcool et aux drogues. Une quatrième limite réside dans le nombre restreint de familles participantes ce qui a eu un impact sur les analyses susceptibles d'être effectuées pour le moment entre les différents profils types.

\section{Références}

Achenbach, T.M. (1991). Manual for the Child Behavior Checklist/4 - 18 and 1991 Profile. Burlington, VT: University of Vermont.

Achenbach, T.M. et Rescorla, L.A. (2001). Manual for the ASEBA School-Age Forms and Profiles. Burlington, VT: University of Vermont, Research Center for Children, Youth and Families.

American Psychiatric Association (2000). Diagnostic and Statistical Manual of Mental Disorders, 4th Edition, Text Revision. Washington, DC: American Psychiatric Publishing.
American Psychiatric Association. (1994). Diagnostic and statistical manual of mental disorders ( $4^{\mathrm{e}}$ éd.). Washington, DC: American Psychiatric Publishing.

Arria, A.M., Mericle, A.A., Meyer, K. et Winters, K.C. (2012). Parental substance use impairment, parenting and substance use disorder risk. Journal of Substance Abuse Treatment, 43, 114-122.

Barnard, M. (2006). Drug addiction and families. London, UK: Jessica Kingsley Publishers.

Barnard M. et McKeganey, N. (2004) The impact of parental drug use on children: What is the problem and what can be done to help? Addiction, 99(5), 552-559. 
Boyer, R., Préville, M., Légaré, G. et Valois, P. (1993). La détresse psychologique dans la population du Québec non institutionnalisée: résultats normatifs de l'enquête Santé Québec. Revue canadienne de psychiatrie, 38, 339-343.

Breton, J.J., Valla, J.P. et Bergeron, L. (1992). Objectifs, pertinence et méthodologie de l'enquête québécoise sur la santé mentale des jeunes de 6 à 14 ans. Santé mentale au Québec, 17, 302-309.

Bukatko, D. et Daehler, M.W. (2004). Child development: A thematic approach (5e éd.). Boston, MA: Houghton Mifflin Company.

Charbonneau R. (2011). Famille et toxicomanie: la problématique $d u$ recrutement (Essai de maîtrise en toxicomanie) Université de Sherbrooke, Québec.

Conners, N.A., Bradley, R.H., Mansell, L.W., Liu, J.Y., Roberts, T.J., Burgdorf, K. et Herrell, J.M. (2004). Children of mothers with serious substance abuse problems: An accumulation of risks. The American Journal of Drug and Alcohol Abuse, 30, 85-100.

Centre de Réadaptation en Dépendance de Montréal-Institut Universitaire (2012). Programme d'intervention auprès des familles d'enfants de 6 à 12 ans touchées par le problème de dépendance. Montréal, Québec.

Crouch, E. C., Bloch, S. et Wanlass, J. (1994). Therapeutic factors: Interpersonal and intrapersonal mechanisms. Dans A. Fuhriman et G. Burlingame (dir.), Handbook of group psychotherapy: An empirical and clinical synthesis (p.269315). New-Jersey, NJ: John Wiley \& Sons.

Dunn, M. G., Tarter, R. E., Mezzich, A. C., Vanyukov, M., Kirisci, L. et Kirillova, G. (2002). Origins and consequences of child neglect in substance abuse families. Clinical Psychology, 22, 10631090.

Epstein, N.B., Baldwin, L.M. et Bishop, D.S. (1983). The McMaster family assessment device. Journal of Marital and Family Therapy, 9, 171-180.
Fals-Stewart, W., Kelley, M.L., Fincham, F.D., Golden, J. et Logsdon, T. (2004). Emotional and behavioral problems of children living with drug-abusing fathers: Comparisons with children living with alcohol-abusing fathers and nonsubstance-abusing fathers. Journal of Family Psychology, 18, 319-330.

Flores, P. (2004). Addiction as an attachment disorder. Lanham, MD: Jason Aronson.

Fraser, J.G., Harris-Britt, A., Thakkallapalli, E.L., Kurtz-Costes, B. et Martin, S. (2010). Emotional availability and psychosocial correlates among mothers in substance-abuse treatment and their young infants. Infant Mental Health Journal, 31, 1-15.

Frick, P.J. (1991). The Alabama Parenting Questionnaire. Manuscrit non publié. Tuscaloosa, AL: University of Alabama.

Groupe de Recherche sur les Inadaptations Sociales de l'Enfance (2003). Guide $d u$ portrait synthèse du jeune et de sa famille. Université de Sherbrooke, Québec.

Hamel, M. (2001). Les relations parentenfants et les pratiques éducatives des parents. In M. Hamel, L. Blanchet et C. Martin (dir.), 6-12-17, nous serons bien mieux!: Les déterminants de la santé et du bien-être des enfants d'âge scolaire (p. 9-49). Sainte-Foy, Québec : Publications du Québec.

Hogan, D. et Higgins, L. (2001) When parents use drugs: Key findings from a study of children in the care of drugusing parents. Dublin: The Children's Research Centre.

Klee H, Jackson M. et Lewis S. (2002). Drug Misuse and Motherhood. London, UK: Routledge.

Koerting, J., Smith, E., Knowles, M. M., Latter, S., Else, H., McCann, $\mathrm{C}$ et al., ... ajouter le dernier auteur (2013). Barriers to, and facilitators of, parenting programmes for childhood behaviour problems: a qualitative synthesis of studies of parents' and professionals' perceptions. European Child \& Adolescent Psychiatry, 22(11), 653-670. 
Kumpfer, K.L. (1997). Strengthening Families Program. Document en ligne: http:// strengtheningfamilies.org $/ \mathrm{html} / \mathrm{model}$ programs_1997/mfp_pg34.html

Kumpfer, K.L. et Bluth, B. (2004). Parent-child transactional processes predictive of resilience or vulnerability to "substance abuse disorders". Substance Use and Misuse, 39, 671-698.

Landry, V., Laventure, M., Gemme, E., Bastien, M-F., Nadeau, C-L., Charbonneau, R.... Bertrand, K. (2010). Toxicomanie parentale et développement des enfants de 6-12 ans: Recension des écrits et pratique de pointe en développement. Les rapports de recherche de l'Institut, Centre Dollard-Cormier - Institut universitaire sur les dépendances.

Laventure, M., Charbonneau, R., Sarrazin, G. et Bertrand, K. (2013). Les programmes d'entraînement aux habiletés parentales: Quand la dépendance et la parentalité se côtoient. Dans C. Plourde, M. Laventure, M. Landry et Arseneault, C. (dir.). Sortir des sentiers battus : Pratiques prometteuses auprès des adultes dépendants.(p. ?) Collection Toxicomanie: Presses de I'Université Laval.

Lussier, K., Laventure, M. et Bertrand, K. (2010). Parenting and Maternal Substance Addiction: Factors Affecting Utilization of Child Protective Services. Substance Use and Misuse, 45, 15721588.

Marsden, J., Ogborne, A., Farrel, M. et Rush, B. (2000). International guidelines for the evaluation of treatment services and systems for psychoactive use disorders. World Health Organisation.

McCubbin, H. et Patterson, J. (1983). Stress: Family inventory of life events and changes. Dans E. Filsinger (dir.), A sourcebook of marriage and family assessment. Beverly Hills, CA: Sage.

Mytton, J., Ingram, J., Manns, S. et Thomas, J. (2014). Facilitators and barriers to engagement in parenting programs: A qualitative systematic review. Health Education \& Behavior, 41(2), 127-137.
Pleck, J. H. (2010). Patemal involvement: Revised conceptualization and theoretical linkages with child outcomes. Dans M. E. Lamb (dir.), The role of the father in child development (5 $5^{\text {ème }}$ éd.) (p. 58-93). Hoboken, NJ: John Wiley \& Sons Inc. ( ${ }^{\circledR}$ éd.1976).

Poole, N. et Dell, C.A. (2005). Toxicomanie au féminin. Centre d'excellence de la Colombie-Britannique pour la santé des femmes et Centre canadien de lutte contre l'alcoolisme et les toxicomanies, Ottawa.

Préville, M., Boyer, R., Potvin, L., Perrault, C. et Légaré, G. (1992). La détresse psychologique: Détermination de la fiabilité et de la validité de la mesure utilisée dans l'Enquête Santé Québec. Ministère de la Santé et des Services Sociaux, Québec

Rossi, H.P., Lipsey, M.W. et Freeman, H.E. (2003). Evaluation: A systematic approach ( $7^{\text {ème }}$ éd.) London, UK: Sage Publications.

Rowland, V.T., Richard, A.D. et Sharon, Y.N. (1985). Perceived adequacy of resources: Development of a scale. Family and Consumer Sciences Research Journal, 14, 218-25.

Scaife, V.H (2008). Maternal and paternal drug misuse and outcomes for children: Identifying risk and protective factors. Children and Society, 22, 53-62.

Shelton, K. K., Frick, P. J. et Wootton, J. (1996). Assessment of parenting practices in families of elementary school-age children. Journal of Clinical Child Psychology, 25(3), 317-329.

Söderström, K. et Skårderud, F. (2013). The Good, the Bad, and the Invisible father: A phenomenological study of fatherhood in men with substance use disorder. Fathering, 11, 31-52.

Solis, J.M., Shadur, J.M., Burns, A.R. et Hussong, A.M. (2013). Understanding the diverse needs of children whose parents abuse substances. Current Drug Abuse reviews, 5, 135-147. 
Street, K., Whitlingum, T., Gibson, P., Cairns, P. et Ellis, M. (2008). Is adequate parenting compatible with maternal drug use? A 5-Year follow-up. Child: Care, Health and Development, 34, 204.

Sword, W., Jack, S., Niccols, A., Milligan, K., Henderson, J. et Thabane, L. (2009). Integrated programs for women with substance use issues and their children: A qualitative meta-synthesis of processes and outcomes. Harm Reduction Journal, 6 (32), 1-17.

Toupin J. et Pauzé, R. (2003): Guide du portrait synthèse du jeune et de sa famille, Groupe de recherche sur les inadaptations sociales de l'enfance, Université de Sherbrooke.

Venne, P. et Morissette, M. (2009). Parentalité, alcool et drogues: Un défi multidisciplinaire. Montréal : Éditions du CHU Ste-Justine.
Vidal S.I., Vandeleur, C., Rothen, S., GholamRezaee, M., Castelao, E., Halfon, O., ...Aubry, J-M., Ferrero, F. et Preisig, M. (2012). Risk of mental disorders in children of parents with alcohol or heroin dependance: A controlled high-risk study. European Addiction Research, 18, 253-264.

Vitaro, F., Assaad, J.M. et Carbonneau, R. (2004). Les enfants de parents affectés d'une dépendance: Bilan des connaissances et leçons pour l'intervention. Montréal, Québec: Comité permanent de lutte à la toxicomanie.

Yalom, I. D. et Leszcz, M. (2005). Theory and practice of group psychotherapy (5 ${ }^{\text {ème }}$ éd.). New York, NY: Basic books. 